\title{
We Need to Plant More Trees for a Healthier Planet!
}

11 hile many of us are at home and keeping a proper social distance from our friends and neighbours, life goes on for nature. Trees continue to grow and make their environmental contributions, and we can help by planting more of them.

During the 2019 federal election campaign, Prime Minister Justin Trudeau committed to planting two billion trees over the next decade. He described how trees sequester carbon and cool our planet. That promise came at a different time, but in the wake of COVID-19 we have an opportunity to improve our planet's health and revive our stalled economy. Simply put: this is the time to plant new forests.

Millions of Canadians are out of work. Meanwhile, tree nurseries across Canada are growing millions of seedlings. Job-seekers can be trained to plant these seedlings. This is an opportune time to mobilize the labour force, including our youth, with a national tree-planting strategy.

Health professionals have asked us to maintain social distance during this pandemic. Fortunately, tree planters work apart, often keeping a minimum distance of two metres between each other. Planting organizations across Canada prepared procedures identify- ing sanitation rules and protocols for planting crews to reduce the risk of COVID-19 transmission.

Forests Ontario commissioned a study last year on the economic impact of planting trees. The study indicated that the roughly 2.5 million trees we plant annually creates approximately 300 seasonal rural jobs. It also revealed a 3:1 financial return from a GDP perspective.

Trees provide countless social and environmental benefits. Tree blossoms feed bees that pollinate farm crops. Trees shade our homes, lower heating bills and increase property values. Forests soak up excess water, mitigate flooding, reduce soil erosion, and provide homes for wildlife. A walk in the forest can strengthen the immune system and reduce blood pressure, among other health benefits. And some trees will one day become paper, furniture, art, musical instruments and building material for homes and office buildings-all products that we need for our standard of living and daily wellbeing.

Forests Ontario has the practical experience to assist in a nation-wide tree planting program. Forest Recovery Canada, our national tree planting arm, has planted trees in British Columbia, Alberta, Ontario, Quebec, Nova Scotia

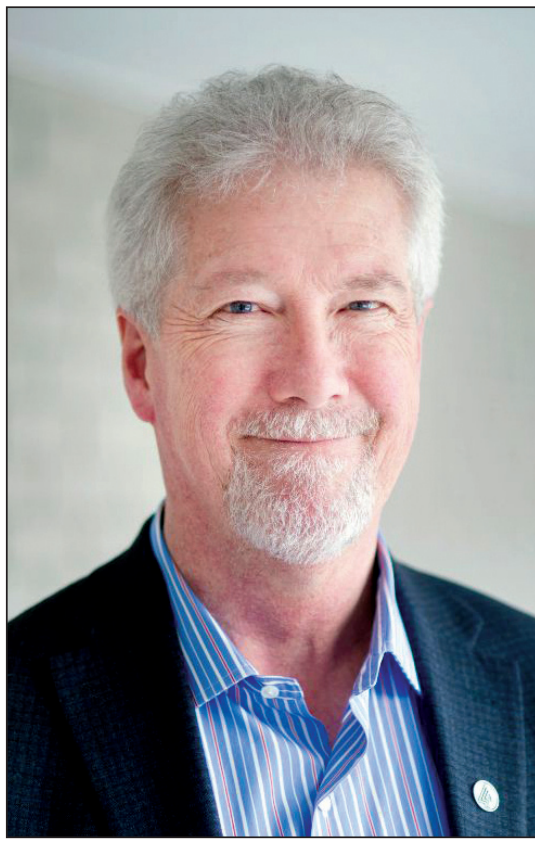

Rob Keen, RPF

rkeen@forests.ontario CEO of Forests Ontario and Forest Recovery Canada/ Président directeur général de Forests Ontario et de Forest Recovery Canada

and Newfoundland and Labrador. And we are the only non-profit organization in Canada that oversees all aspects of forest restoration-from seed to forest.

Let's put Canadians back to work planting trees and strengthen the health of our planet. We are ready to be a part of the solution. 


\section{II nous faut planter plus d'arbres pour améliorer l'état de la planète !}

A lors que nous sommes nombreux à rester à la maison et à appliquer une saine distanciation sociale avec nos amis et nos voisins, la nature poursuit son cours. Les arbres continuent de croître et d'apporter leur contribution environnementale et nous pouvons nous impliquer en plantant plus d'arbres.

Au cours de la campagne électorale fédérale de 2019, le premier ministre Justin Trudeau s'est engagé à planter deux milliards d'arbres au cours de la prochaine décennie. Il a décrit l'importance de la séquestration du carbone par les arbres et leur effet de rafraîchissement des températures. Cette promesse a été faite dans un autre contexte, mais présentement face à la COVID-19, nous avons l'opportunité d'améliorer l'état de la planète et de relancer notre économie au point mort. En d'autres mots : c'est le moment de planter de nouvelles forêts.

Des millions de Canadiens et Canadiennes sont sans travail actuellement. Les pépinières forestières de tout le Canada doivent produire des millions de semis. Les personnes à la recherche d'un emploi peuvent être formé pour planter ces semis. Il s'agit d'une occasion unique nous permettant de mobiliser la main d'œuvre, ainsi que les jeunes, dans le cadre d'une stratégie nationale de plantation d'arbres.
Les autorités sanitaires nous ont demandé de conserver une distanciation sociale au cours de la pandémie. Heureusement, les ouvriers sylvicoles travaillent éloignés les uns des autres, maintenant souvent une distance minimale de 2 mètres entre eux. Les entreprises spécialisées dans la plantation d'arbres de tout le Canada ont élaboré des directives portant sur les mesures sanitaires et les protocoles destinés aux équipes de planteurs afin de réduire le risque de transmission de la COVID-19.

Forests Ontario a commandé une étude l'an dernier sur les impacts économiques de la plantation d'arbres. L'étude a démontré que les 2,5 millions d'arbres que nous plantons chaque année entraîne la création de 300 emplois saisonniers en milieu rural. Elle a également relevé un ratio financier de 3:1 en termes de PIB.

Les arbres procurent une impressionnante quantité des bénéfices sociaux et environnementaux. La floraison des arbres permet de nourrir les abeilles qui pollinisent les récoltes. Les arbres atténuent les ardeurs du soleil sur les maisons, réduisent les factures de chauffage et accroissent la valeur des propriétés. Les forêts absorbent l'eau de ruissellement, réduisent l'impact des inondations, maintiennent le sol en place et constituent un habitat pour la faune.
Une marche en forêt peut renforcer le système immunitaire et réduire la tension artérielle, ainsi qu'apporter d'autres bénéfices en terme de santé. Sans oublier que certains de ces arbres deviendront un jour du papier, des meubles, des sculptures, des instruments de musique et du matériel de construction tant pour des maisons que des édifices à bureaux-tous des produits requis pour maintenir notre niveau de vie et notre bien-être quotidien.

Forets Ontario détient une expertise pratique lui permettant de s'impliquer dans un programme national de plantation d'arbres. Forest Recovery Canada, notre filiale nationale de plantation, a complété des projets en Colombie-Britannique, en Alberta, en Ontario, au Québec, en Nouvelle-Écosse et à TerreNeuve- Labrador. Et nous sommes le seul organisme sans but lucratif au Canada qui supervise tous les aspects du reboisement forestier-de la semence à la forêt.

Aidons les Canadiens et les Canadiennes à retourner au travail par la plantation d'arbres et l'amélioration de l'état de la planète. Nous sommes prêts à être un élément de la solution. 